\title{
Correction to: Pediatric Board Study Guide
}

\author{
Osama I. Naga
}

\section{Correction to: O. I. Naga (ed.), Pediatric Board Study Guide, https://doi.org/10.1007/978-3-030-21267-4}

This book was inadvertently published with a couple of incorrect sentences that have been corrected now.

In Chapter 4, p. 115, the sentence 'Cervical flexion-extension radiographs by 3-5 years, when planning to participate in contact sports or if neck pain, torticollis, gait disturbance, or weakness' has now been corrected to 'Sport pre-participation screening for atlanto-axial instability (AAI) symptoms e.g., neck pain, torticollis, gait disturbance, or weakness. Routine neck X-ray for asymptomatic patients with DS is no longer recommended.'

In Chapter 6, the phrase "Dry mouth" has been removed from Table 6.4 (last row, column 4).

In Chapter 14, p. 510, the sentence "The Special Olympics organization requires radiographic evaluation of the cervical spine before sports participation' has now been corrected to 'Sport pre-participation screening for AAI symptoms. Routine neck $\mathrm{x}$-ray for asymptomatic patients is no longer recommended.'

The updated versions of the chapters can be found at https://doi.org/10.1007/978-3-030-21267-4_4 https://doi.org/10.1007/978-3-030-21267-4_6 https://doi.org/10.1007/978-3-030-21267-4_14 